\title{
Design of a Low Energy Electron Impact Spectrometer
}

\author{
Adrian Swanson and Sanford Lipsky \\ University of Minne sota \\ Department of Chemistry \\ Minneapolis, Minnesota 55455
}

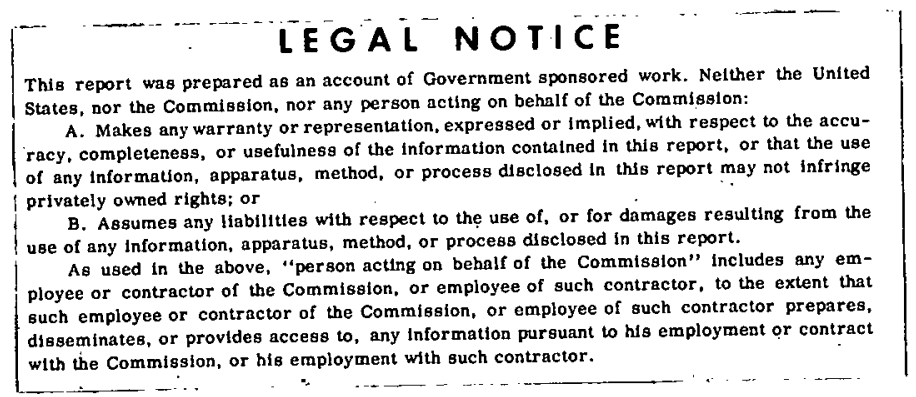




\section{DISCLAIMER}

This report was prepared as an account of work sponsored by an agency of the United States Government. Neither the United States Government nor any agency Thereof, nor any of their employees, makes any warranty, express or implied, or assumes any legal liability or responsibility for the accuracy, completeness, or usefulness of any information, apparatus, product, or process disclosed, or represents that its use would not infringe privately owned rights. Reference herein to any specific commercial product, process, or service by trade name, trademark, manufacturer, or otherwise does not necessarily constitute or imply its endorsement, recommendation, or favoring by the United States Government or any agency thereof. The views and opinions of authors expressed herein do not necessarily state or reflect those of the United States Government or any agency thereof. 


\section{DISCLAIMER}

Portions of this document may be illegible in electronic image products. Images are produced from the best available original document. 


\section{INTRODUCTION}

The efforts of the past year (1968-69) have been mainly directed towards mastering the design elements of a low energy electron impact spectrometer. Using computer assisted techniques, a theoretically successful electron-optical configuration has been arrived at and we are now ready to begin construction of the actual spectrometer.

The purpose of this spectrometer is to identify low lying triplet states of polyatomic molecules and to study the threshold behavior of their scattering cross sections. The physical phenomenon observed is the energy loss suffered by a nearly monoenergetic beam of electrons incident on a static target gas. The target gas is confined to a small scattering chamber at the center of the spectrometer into which the incident beam is projected. Two electron energy analyzers are mounted adjacent to the scattering chamber and have their entrance apertures oriented at 0 degrees and 90 degrees with respect to the incident beam. Figure 1 illustrates the physical relationship of the various spectrometer components.

The entire spectrometer is housed in a mercury pumped, cold trapped, bakeable, ultra-high vacuum chamber, shown schematically in Figure 2. Internal radiant heaters will maintain uniform spectrometer and scattering chamber temperatures up to about 150 degrees centigrade during operation.

The basic structure and the design philosophy of the spectrometer follows that of Kuyatt and Simpson ${ }^{1,2,3}$ with the incorporation of four new features. The first of these is the simultaneous use of two analyzers placed at 0 and 90 degrees.

J.A. Simpson, Rev. Sci. Instr. 35, 1698 (1964).

${ }^{2}$ C.E. Kuyatt and J.A. Simpson, Rev. Sci. Instr. 38, 103 (1967). 3 Personal communication. 
Little is yet known about the angular dependence of the differential crosssection for a transition involving change in spin multiplicity. Nevertheless, the effective range of the potential for such a transition is expected to be generally smaller than for an optically allowed transition, predicting, therefore, a more spherically symmetrical scattered electron distribution. Since for many of the molecules that will be initially studied, the singlet and triplet transition energies tend to overlap, with the singlet transition generally dominating the cross section, the ability to simultaneously monitor the beam at two angles should aid considerably in resolving the triplet transition.

A second feature is the projection of an approximately 'quasi-parallel' beam into the scattering chamber by the monochromator. 'Quasi-parallel' here refers to a beam which is confined over a short distance to a cylindrical volume of space, although individual electron trajectories are divergent from the central axis. The major advantage of a quasi-parallel beam is that, when properly focused, it is not attenuated by the scattering chamber apertures and therefore zero angle scattered electrons are not lost to the analyzer. The quasi-parallel property also is expected to aid in alignment of the spectrometer because, when properly tuned, a plateau in the transmitted current signals the fact that the beam is easily clearing the scattering chamber apertures.

The third innovation concerns the manner in which scanning the spectrometer is accomplished. As the incident beam energy is varied and/or the energy loss bandpass of the analyzer is varied, the electron-optical systems are continuously refocused to maintain constant resolution and transmission properties. 
The fourth feature is the proximate connection of a capacitance manometer directly to the scattering chamber. The manometer will be mounted inside the vacuum system and maintained at the temperature of the scattering chamber. The rationale behind this is to eliminate thermal transpiration effects in measuring target gas concentrations. This ensures accurate determinations of the scattering cross section dependence on pressure.

In the following sections these and other general features of the present spectrometer design will be presented in more detail. 


\section{THE MONOCHROMATOR}

The function of the monochromator is to project a beam of nearly monoenergetic electrons into the scattering chamber. The design center properties of the beam are:

$$
\begin{aligned}
& \text { Mean Beam Energy: } 1 \text { to } 10 \text { electron volts } \\
& \text { Energy Spread: } \quad \text { " } 0.050 \text { e.v. (F.W.H.M.) } \\
& \text { Scattering Chämber } \\
& \text { Beam Profile: Quasi-parallel with maximum beam } \\
& \text { diameter less than } 50 \mathrm{mils}
\end{aligned}
$$

Using the methods of Gaussian dioptrics and the general procedures of Simpson and Kuyatt ${ }^{1,2}$ a monochromator has been designed which satisfies the above criteria. The theoretically expected maximum monochromator output current at the above resolution is of the order of 0.1 microampere. The monochromator is schematically indicated in Figure 3 and a detailed drawing is given in Figure 4. The overall scheme is this: The diode gun and condenser lens 'illuminate' the input apertures of the remaining optical train with electrons. The 10:1 decellerating lens, the spherical deflector and the 1:10 accelerating lens combination comprise the actual energy dispersing eliement of the monochromator. The field lens acts to control the pupil position of the beam at the scattering chamber while the focus lens controls the window position. The lens spacings and diameters control the magnification properties of the optical train. Computer programs supplied by Kuyatt were used to synthesize and evaluate the various electron lens systems. 
III. THE DIODE GUN AND CONDENSER IENS

The diode gun, Figure 5 , is the actual source of electrons. It consists of a dispenser type of indirectly heated cathode positioned with its. face parallel to a thin anode plate having a small circular aperture on the axis of symmetry. A positive potential is applied to the anode and a Pierce element surrounds the cathode to maintain a uniform electric field normal to the cathode face. This combination results in an immersion type of Calbik lens whose focal properties are largely independent of the applied voltage ratio. Emission from the cathode is space charge limited and therefore the beam current emerging from the diode gun aperture is proportional to the $3 / 2$ 's power of the applied voltage. Thus the diode gun produces an electron beam whose geometry is fixed by the gun dimensions and which has a current density which increases with anode potential.

The condenser lens element serves to relay the electron beam from the diode gun to the apertures of the 10:1 decelerater lens. The magnification of the condenser is chosen such that these apertures are 'over-filled' with electrons, thus ensuring that they alone determine the initial window and pupil of the beam. 


\section{THE ENERGY DISPERSING AND RESOLVING EIEMENTS}

The theory of spherical energy filters using virtual apertures is well discussed in Reference 2. For an energy resolution of $0.050 \mathrm{e.v}$. and a one inch nominal sphere radius, the optimum median sphere energy is predicted to be 5.0 volt. Figure 6 illustrates the arrangement of the energy dispersing elements. Real slits are not used at the faces of the sphere gap to delimit the electron trajectories since their presence would not only distort the radial symmetry of the electric field but secondary electrons produced at the slit edges would be propagated along with the primary beam and appear at the output of the monochromator as an undesirable broadening of the beam energy spread. Instead, the initial window aperture is imaged onto the face of the sphere gap entrance by means of a 10:1 decelerating lens. The initial pupil aperture is positioned at the first focal point of this lens and therefore the beam enters the sphere gap with its pupil at infinity. Because the faces of the sphere gaps have the property of first order unit planes, the emergent, energy-dispersed beam has its pupil still at infinity, but the window now is located at the exit face of the sphere gap. A 1:10 accelerating lens with a geometry identical to the $10: 1$ lens images the dispersed beam onto a real slit which intercepts : alï but those electrons having a kinetic energy ten times greater than the median sphere potential, i.e., 50 electron volts. This slit has the same width as the initial window aperture so no loss of $50.0 \pm 0.025 \mathrm{e} \cdot \mathrm{v}$. electrons occurs and hence the slit is identical with the beam window. (A slit is used here rather than a circular aperture to accommodate any lateral misalignment of the sphere and the axis of the monochromator input and output legs. Any misalignment will be compensated later in the optical train by a set of 'steering' deflectors.) As the beam leaves the 10:1 lens the pupil is at the second focal point and has the same diameter as the initial pupil aperture. 
V. THE FIELD AND FOCUS LENSES

The field and focus lenses perform the function of relaying the electron beam to the scattering chamber and maintaining control over the window and pupil positions as the beam energy is varied. Their function is to place the beam window to the right of the scattering chamber and

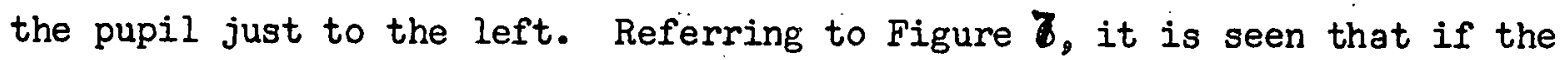
window and pupil diameters are the same, a quasi-parallel beam is produced over the length of the scattering chamber.

The field lens acts to decouple the pupil and window positions. This is accomplished by choosing the geometry of the field lens such that the beam pupil lies close to the first focal point of the lens while the window is as close as possible to the lens center. This arrangement moves the pupil towards infinity without disturbing the window size and position to any great extent as is shown in Figure 8.

The focus lens is actually a compound lens and its action is shown in Figure 8 . There are two regions of lens action, one between the field lens element and the focus element and one between the focus element and the scattering chamber element, but since their field gradients do not overlap, their overall effect may be regarded as being concentrated in a single virtual lens located between the two real lenses.

In the course of operation one will wish to vary the scattering chamber potential (i.e., the incident beam energy) without disturbing the quasiparallel beam feature. How this can be done may be seen from the following considerations. The focal lengths of the compound lens are strictly proportional to the square root of the input and output leg potentials respectively, while the positions of the principal planes are moved left or right 
as the potential of the focus element is varied. The spacing between the principal planes depends mainly on the spacings between the real lenses and to a lesser extent on the focus element potential. Thus as the output lens potential (i.e., the scattering chamber potential) is varied and the output focal length changes as a result, the position of the principal planes are changed by adjusting the focus potential to keep the window position constant. The window magnification remains nearly constant since the effective lens center does not move much as the potentials are varied. The pupil position of the output beam falls near the second focal position of the compound lens because its position was near infinity as the beam. entered the lens. Since the pupil position moves as the potentials are varied, so also does its magnification. The beam profile at several scattering chamber potentials is shown in Figure 10. 


\section{THE SCATTERING CHAMBER}

The detail of the scattering chamber is shown in Figure "20. Its function is to confine the target gas to as small a volume as possible. The target gas enters at the sile through a viscous leak with a conductance of about $5 \mathrm{cc} / \mathrm{sec}$ and leaves via the three beam apertures. The beam apertures are kept as small as practical in order to maintaln a high differential pressure between the scattering chamber and the rest of the vacuum system. This will minimize degradation of the beam properties in the monochromator and analyzers. An equilibrium gas concentration is maintained by balancing the input and output flow rates. The input flow rate is controlled by varying the gas pressure on the reservoir side of the leak, whereas the output conductance remains relatively constant with pressure changes in the scattering chamber.

The target gas pressure is measured with a precision capacitance manometer attached directly to and kept at the same temperature as the scattering chamber. This refinement, not incorporated into earlier electron impact spectrometers, is necessary if multiple scattering events are to be identified. Experimentally one varies the target gas pressure and observes its effect on the scattering cross sections. It is not sufficient to monitor the pressure changes as a function of the reservoir pressure. The target gas pressures are such that the gas transport properties in the scattering chamber are characteristic of Knudsen flow, so that the equilibrium pressure will not depend linearly on the input flow rate. For the same reasons the background pressure of the vacuum chamber cannot be used to estimate the target gas concentration which therefore must be measured directly. 
If thermal transpiration effects are to be avoided in the direct measurement process, then the scattering chamber, the pressure measuring device and their connecting link must all be at the same temperature or else the dimensions of the link must be large compared to the mean free path of the gas. As the latter is not feasible, the former condition must be satisfied since corrections for thermal transpiration effects are impossible under Knudsen flow conditions.

In order to maximize the pressure differential between the scattering chamber and the vacuum chamber, the area available to the gas for escape must be a minimum, i.e., the gas escapes only through the beam apertures. This is an unfortunate situation since it means that the gas concentration outside the scattering chamber will be greatest at the opening of the tubular electron lenses, resulting in a preferential diffusion of gas down the optical train, exactly where we would wish to minimize the number of electron-molecule interactions. To combat this diffusion of gas, two approaches will be tried (see Figure 11). First, placement of a double aperture at the scattering chamber may provide a degree of differential pumping of the effluent gas and, secondly, the lens elements will have numerous vent ports drilled obliquely through their wall to enhance the escape of gas from the lens axis. 
VII. THE ENERGY ANAIYZERS

The electron-optical design of the two analyzers is identical. The 90 degree analyzer will therefore be discussed in what follows, since it differs only in that an electron multiplier is used as a detector, whereas a Faraday cage is the detector in the zero degree analyzer.

The function of the energy analyzer is to collect electrons scattered from the beam by the target gas and determine their kinetic energy distribution. The following are the design center values for the analyzer:

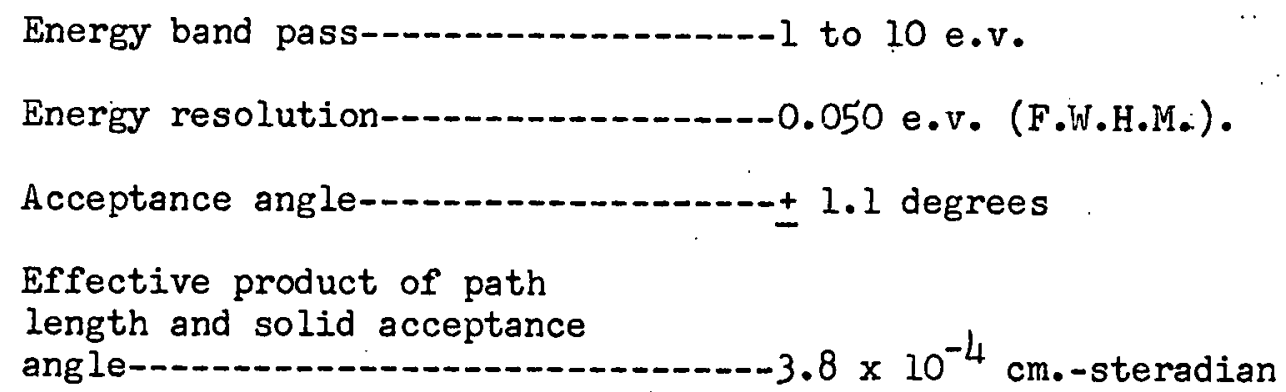

The geometry of the analyzer input apertures with respect to the incident beam is shown in Figure 13. Note that the window of the scattered electrons is naturally limited in the lateral direction by the incident beam width but must be limited in the axial direction by an extra slit in order to retain a cylindrically symmetric beam in the analyzer. The slit width is chosen such that the axial and lateral window dimensions are equal and the height is such as to eliminate the possibility of vignetting the lateral window due to misalignment of the pupil aperture and the beam.

A schematic of the analyzer is shown in Figure 14. The design philosophy is similar to that of the monochromator stage. The field lens decouples the window and pupil positions for the focus lens. The focus lens is again a compound lens which relays the scattered beam to the input apertures of the analyzing sphere system. The operation of the spheres is the 
same as in the monochromator except that no attempt is made to place the pupil at infinity as the beam enters the sphere gap. The criterion here is not to control the pupil position, but rather to avoid beam spreading to the surfaces of the hemispheres, thereby attenuating the beam. and reducing the detection sensitivity.

The Soa lens in Figure 15 is a type of triode lens which accelerates, collimates and projects the energy resolved beam onto the detector. 


\section{SPECTRONETER SCANNING MODES}

There are two different modes of scanning the spectrometer. In the first, or energy loss mode, the incident beam energy is kept fixed while the energy band pass of the analyzers is changed. In the designs of Kuyatt and Simpson ${ }^{1,2}$ this type of scanning is accomplished by means of an 'energyadd' lens scheme wherein the energy lost by the electrons in an inelastic scattering encounter is restored by adding a fixed voltage to each of the elements of the analyzer. This preserves the effective voltage ratios of all the electron lenses (and therefore their optical properties) with the exception of the field lens. If the field lens is properly designed, its change in focusing power can be made to have a minimum effect on the analyzer resolution and transmission. For incident beam energies greater than about 20 volts this minimum effect is negligible but at 10 volts and less it becomes intolerable. Therefore, in the present instrument it will be necessary to refocus the field and focus lenses in conjunction with the addition of the 'energy-loss' voltage.

In general the required refocusing potentials are not linear functions of the energy loss voltage and since it is very desirable to scan the energy loss spectrum with a linear ramp function as the horizontal ordinate of an $X-Y$ recorder, some adjustable, non-linear voltage supply is required which can be driven by the ramp function. Such a nonlinear supply is formed from a diode function generator, an operational amplifier and voltage programable power supply as indicated in Figure 16.

In the second mode of scanning the incident beam energy is varied while the analyzer band pass is kept at some fixed energy loss. In this mode not only must the analyzers be refocussed but so also must be the monochromator if the quasi-parallel beam is to be preserved. A block diagram of the scanning system is shown in Figure 17. 


\section{MECHANICAL CONSIDERATIONS}

The manner in which the lens elements and hemispheres are assembled is shown in Figure 18. Three polished, precision glass balls insulate and support adjacent lens elements. Each leg of an electron optical system is held together by means of three threaded stainless steel rods with nuts. A precisely machined and stress relieved stainless steel (type 304) plate is the sole support for the lens elements and hemispheres. The monochromator and the two analyzers are each mounted on such a plate which in turn is fixed at right angles to a large base plate. The scattering chamber is also fastened to this plate and all four can be independently positioned with respect to one another. This arrangement allows considerable flexibility in accommodating modifications to the spectrometer and future designs. 
Note:

Spherical elements shown as tubes for clarity

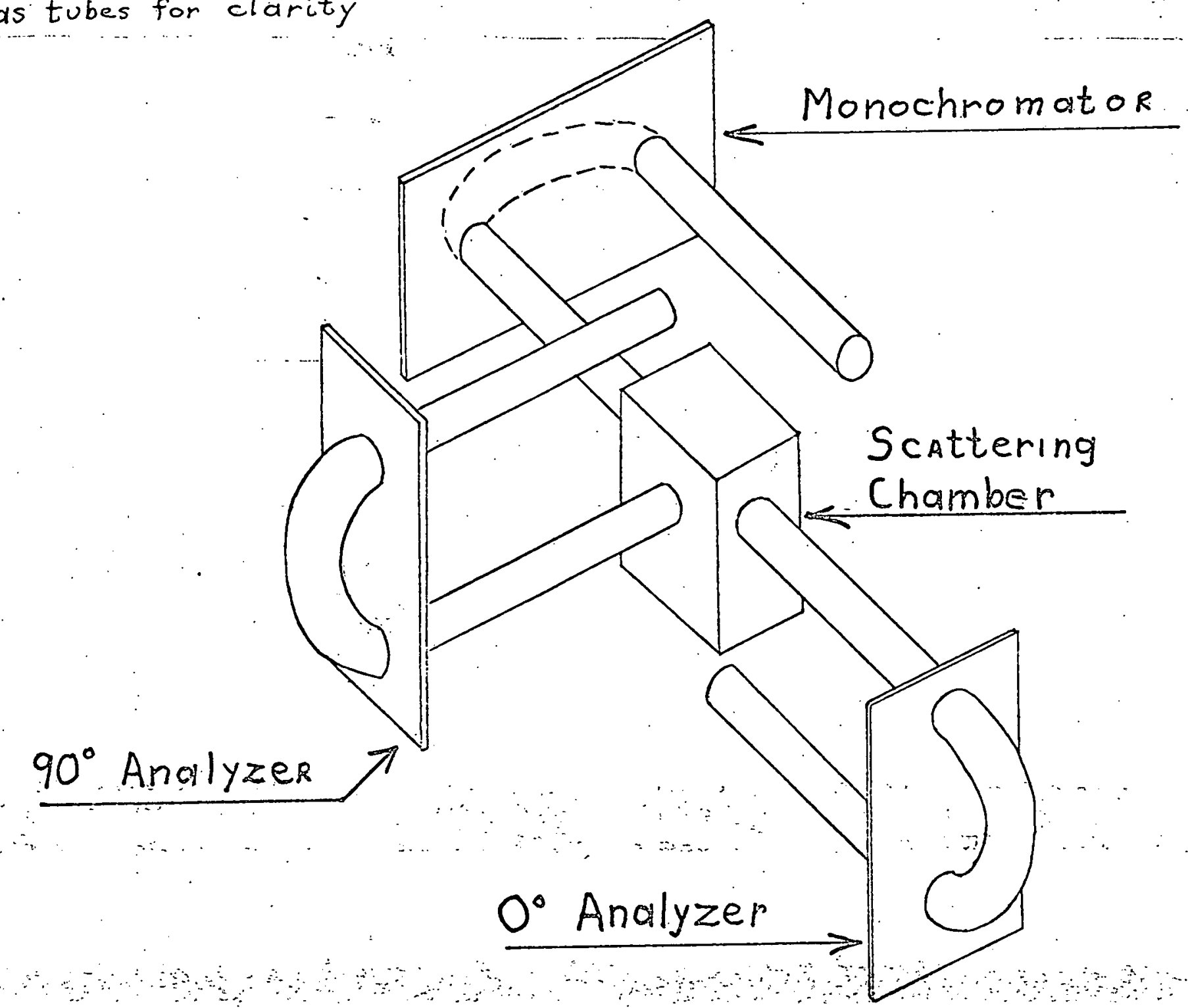

Fig. 1 


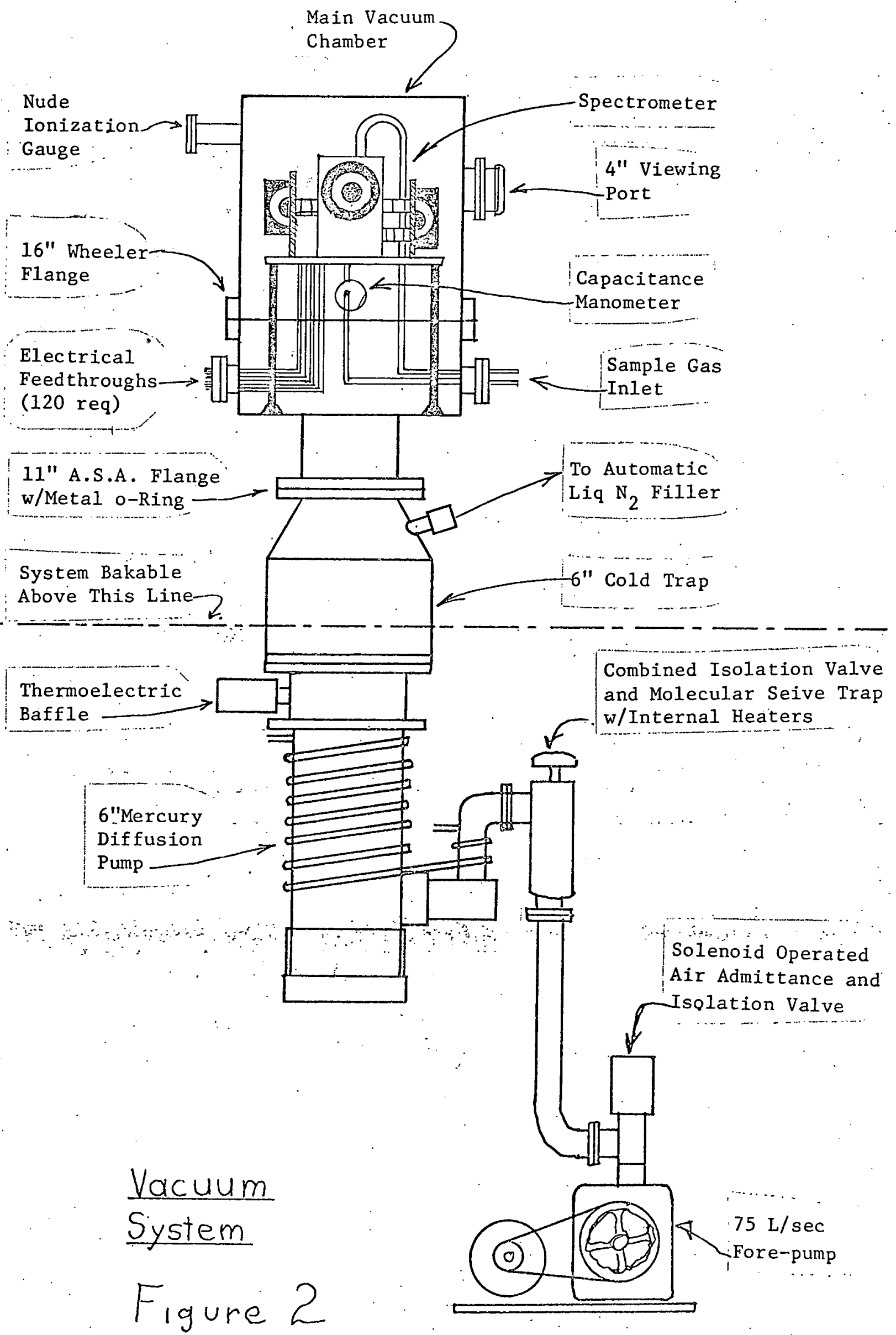




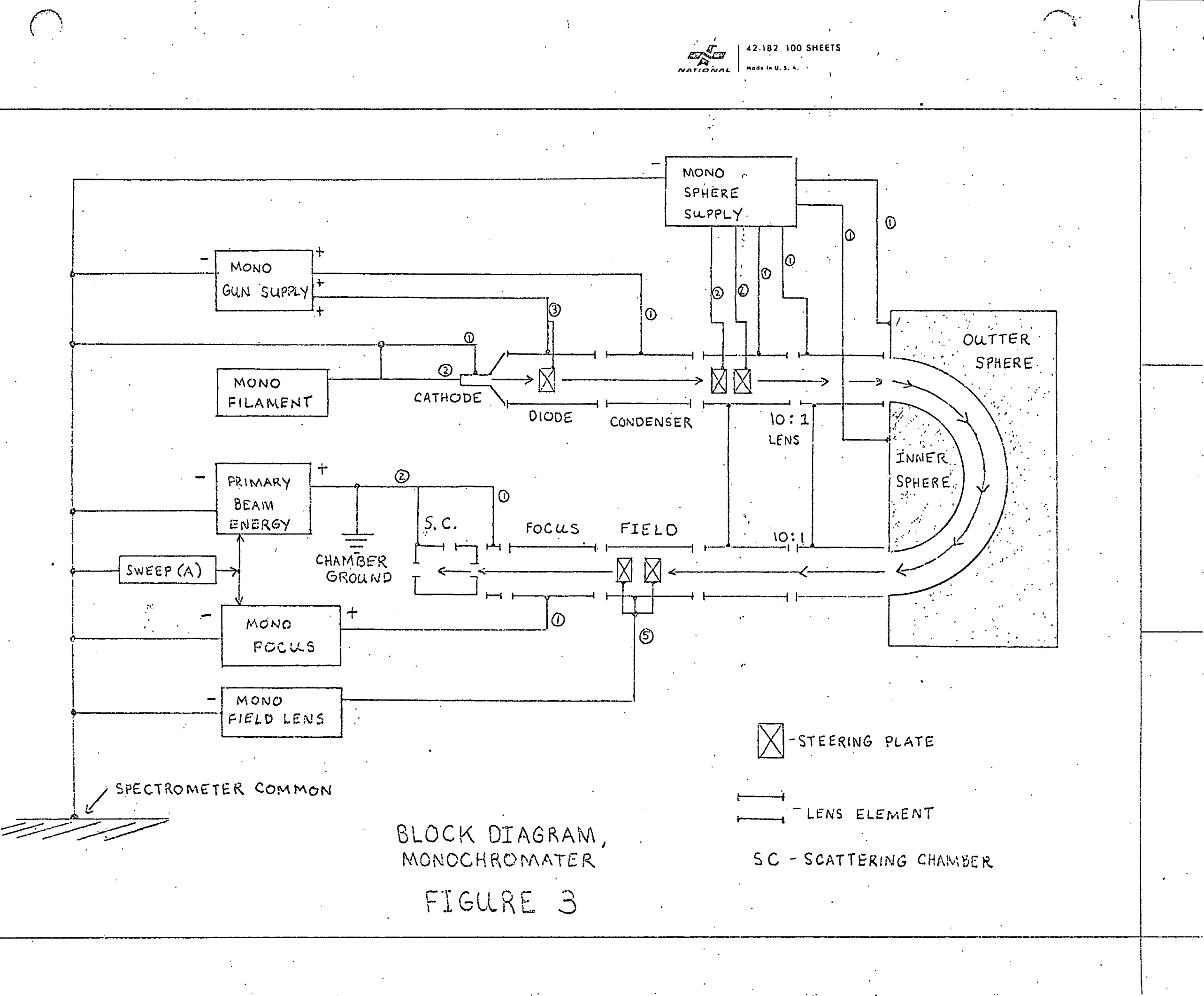




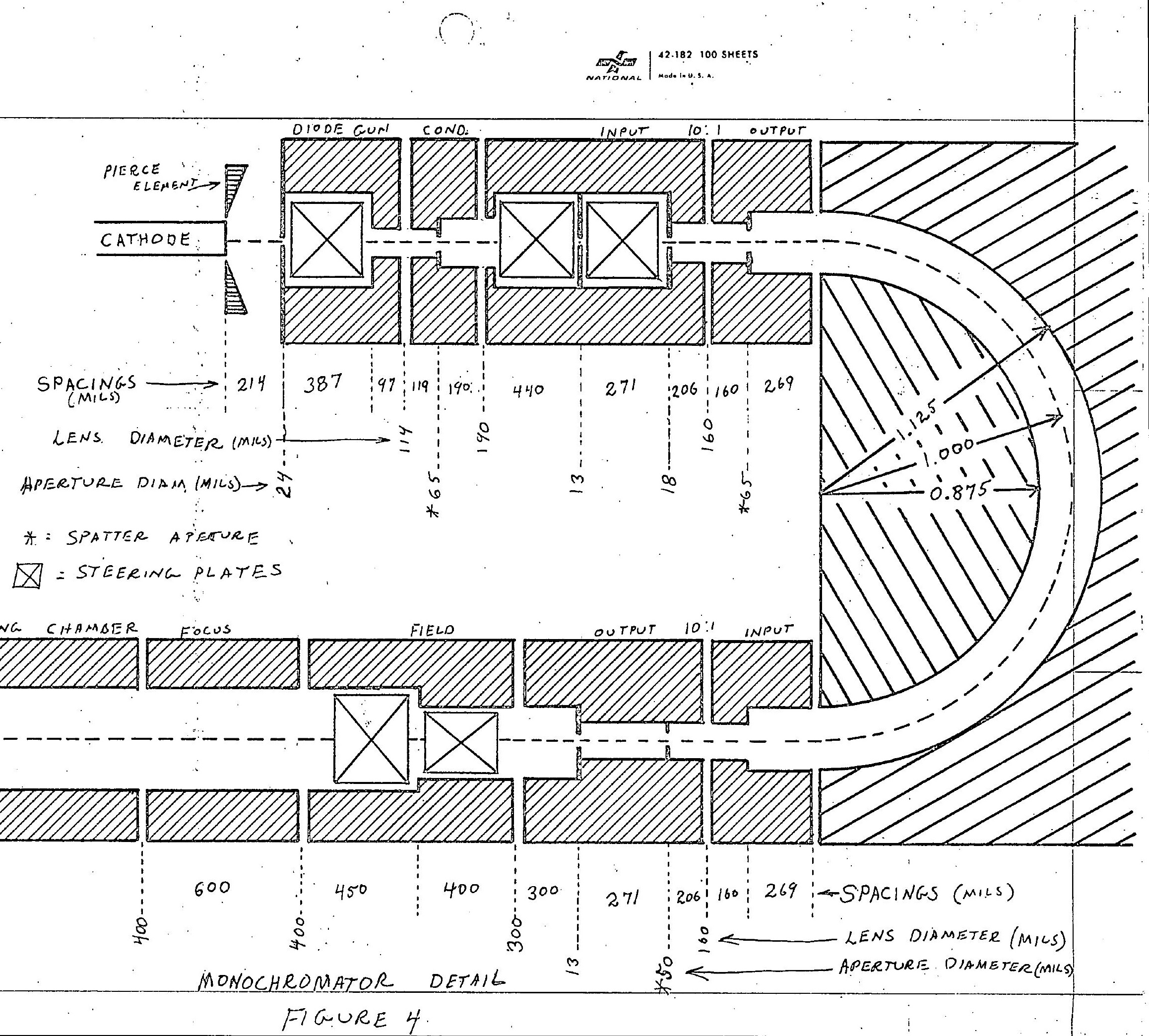




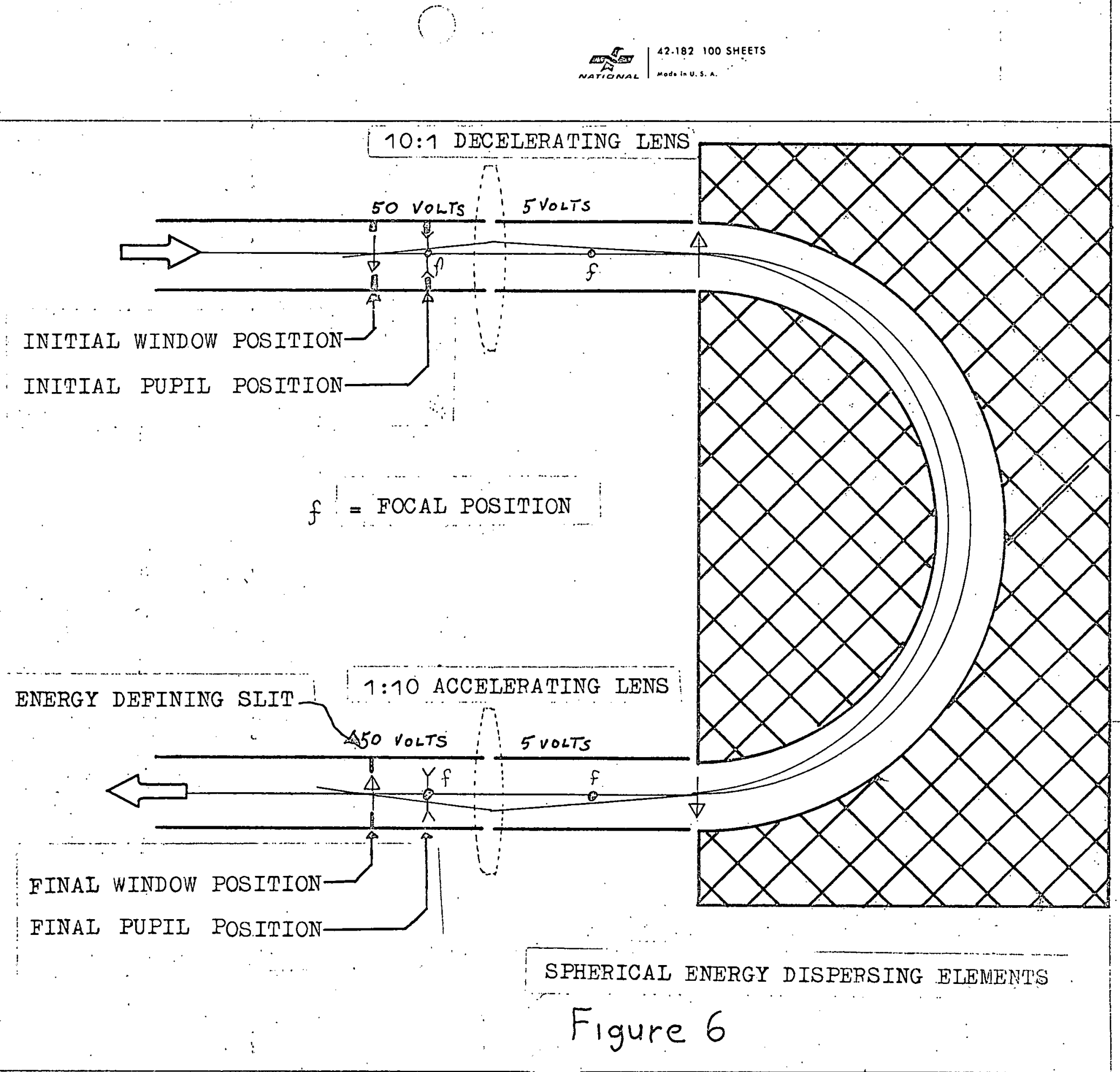




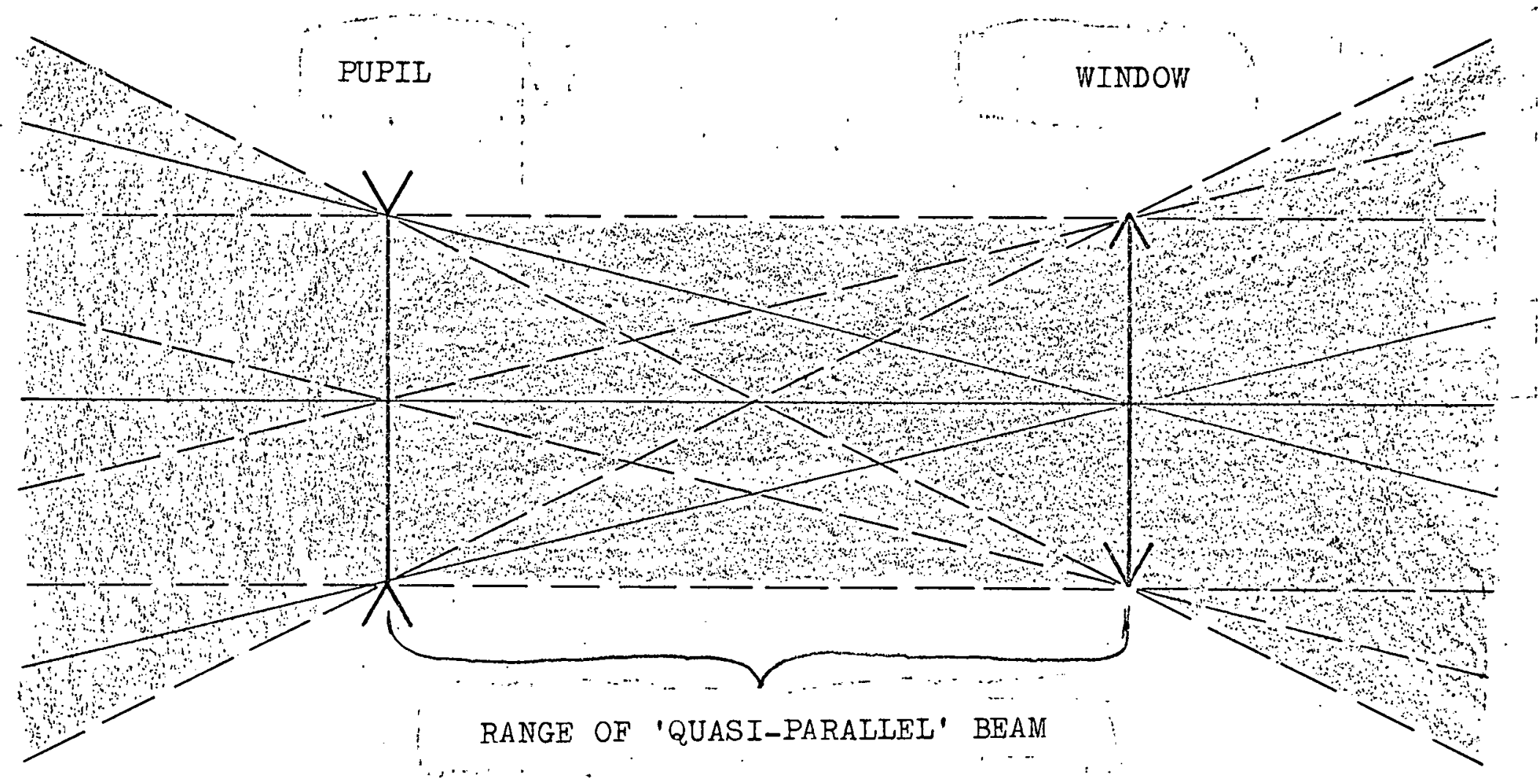

Figure 7 


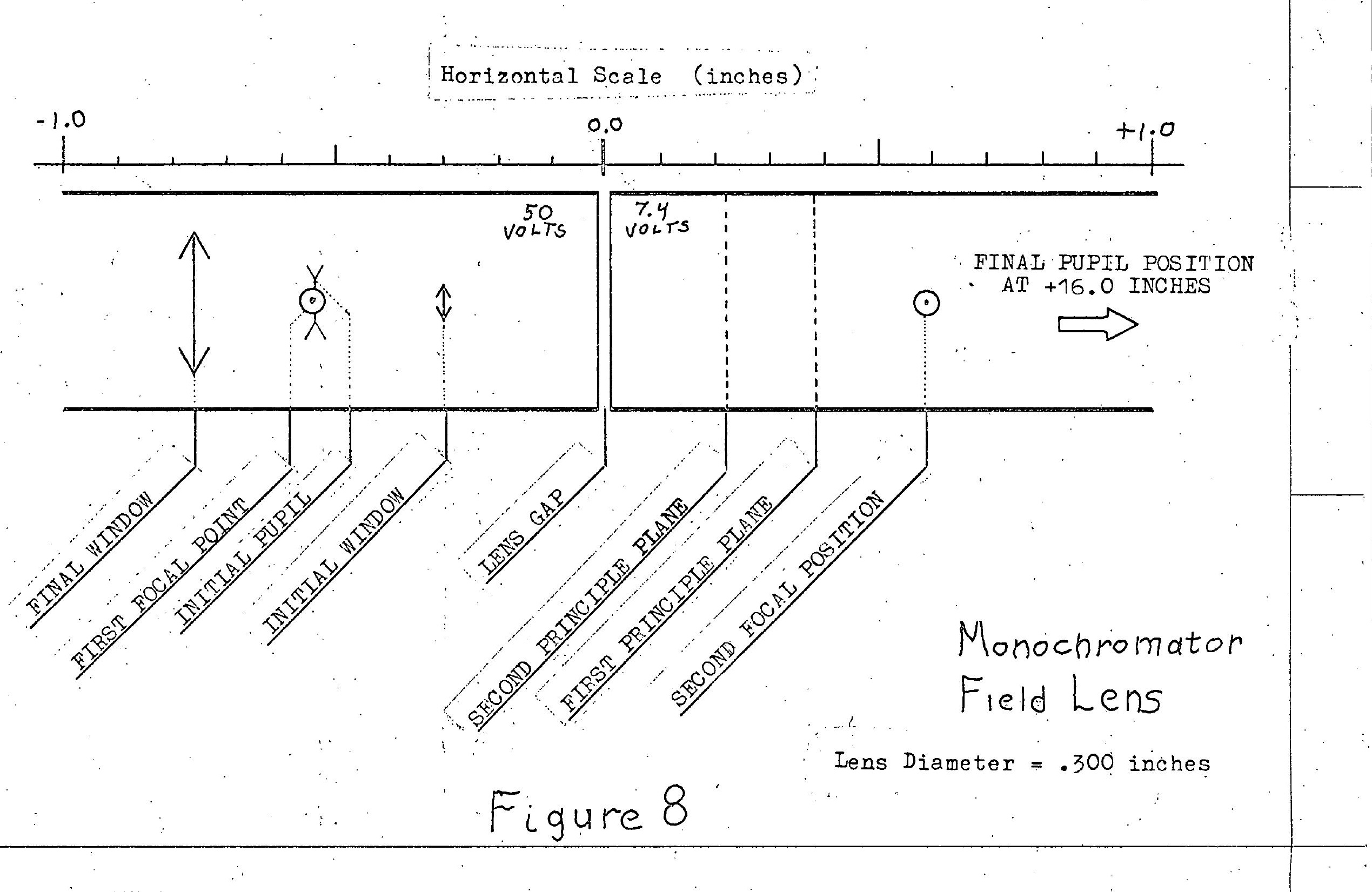




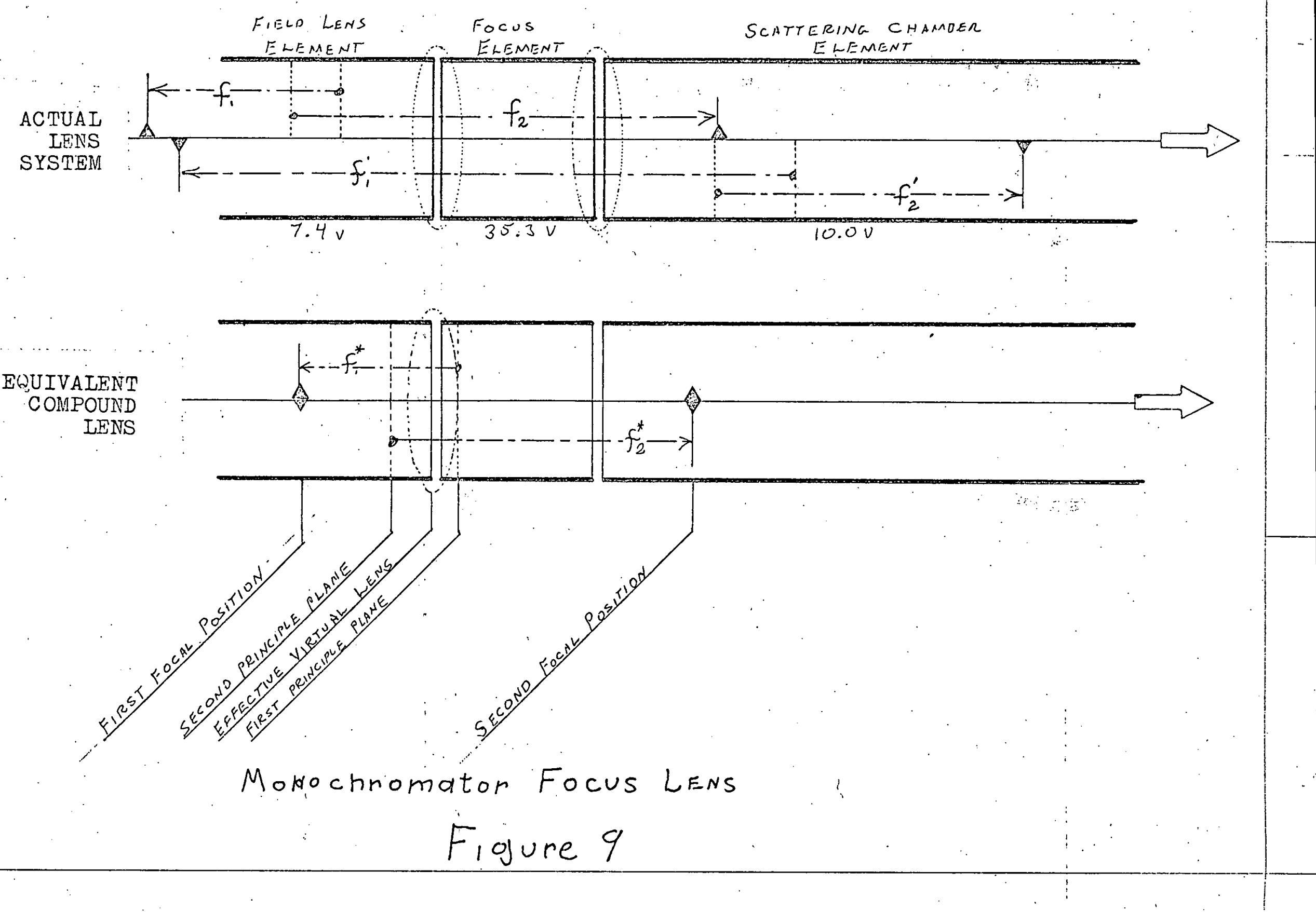




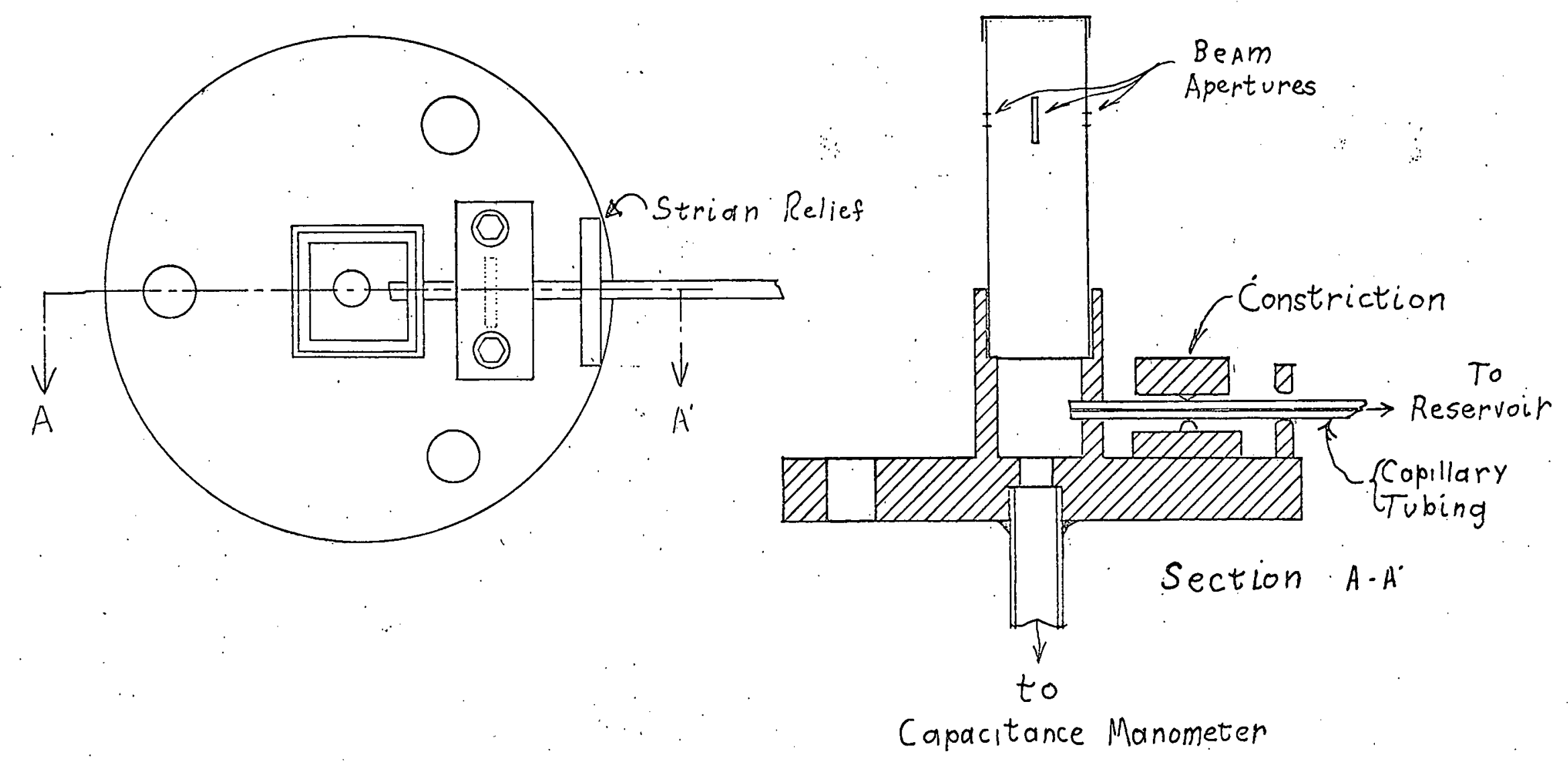

Figure 11

Scattering Chamber 
Monochromator

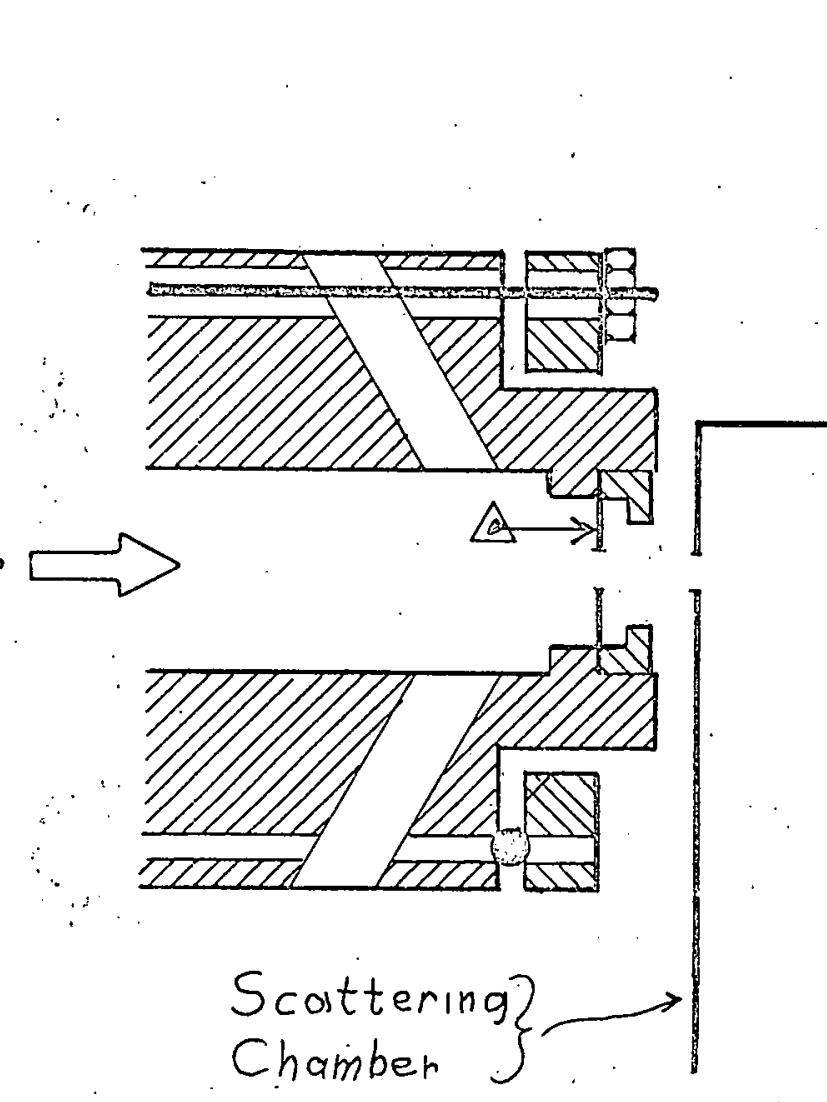

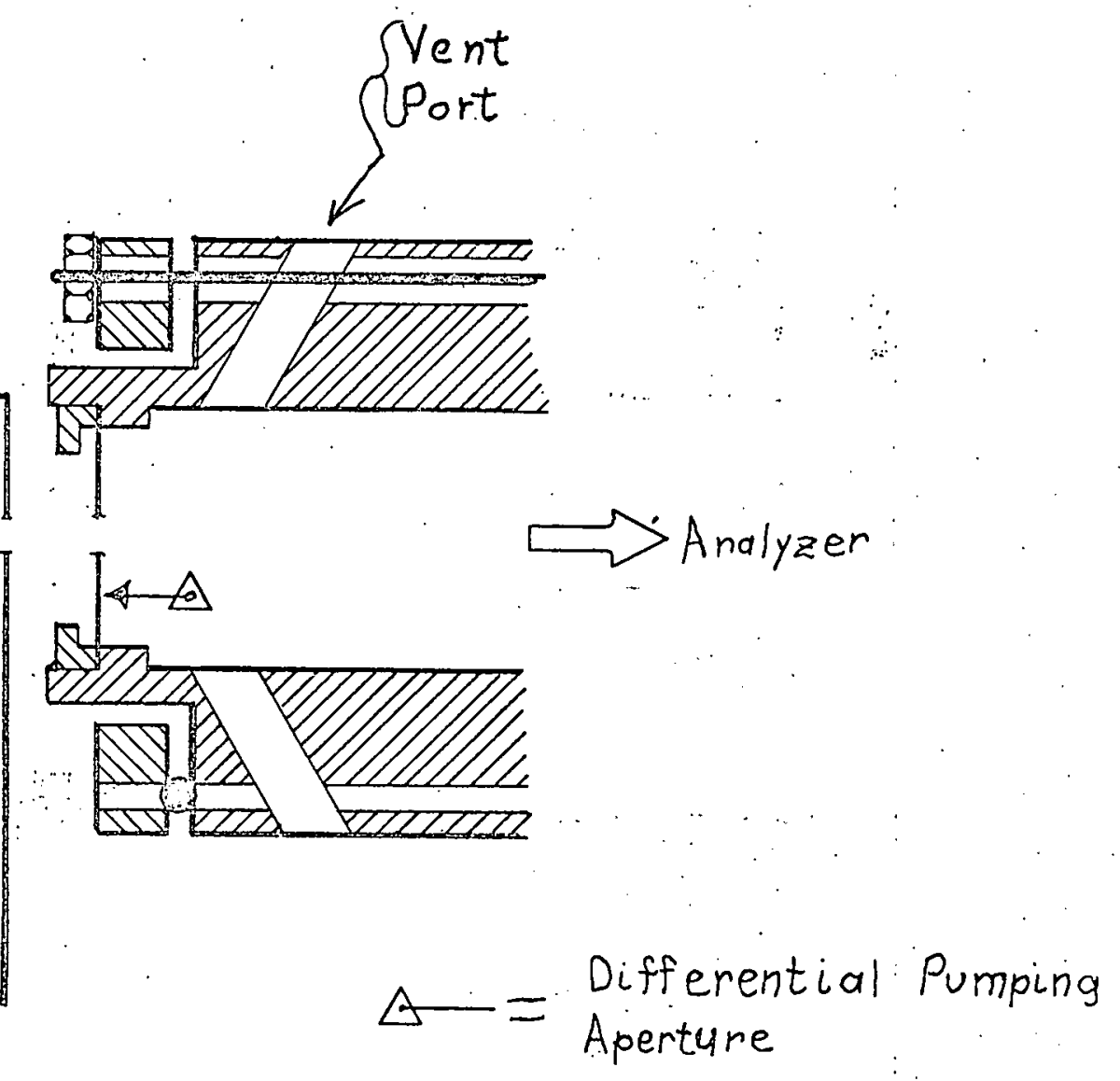

Differential Pumping of the Scattering Chamber

Figure 12 


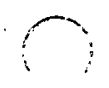

40

42.182 100 SHEETS

NOTE:

Not Drawn to Scale
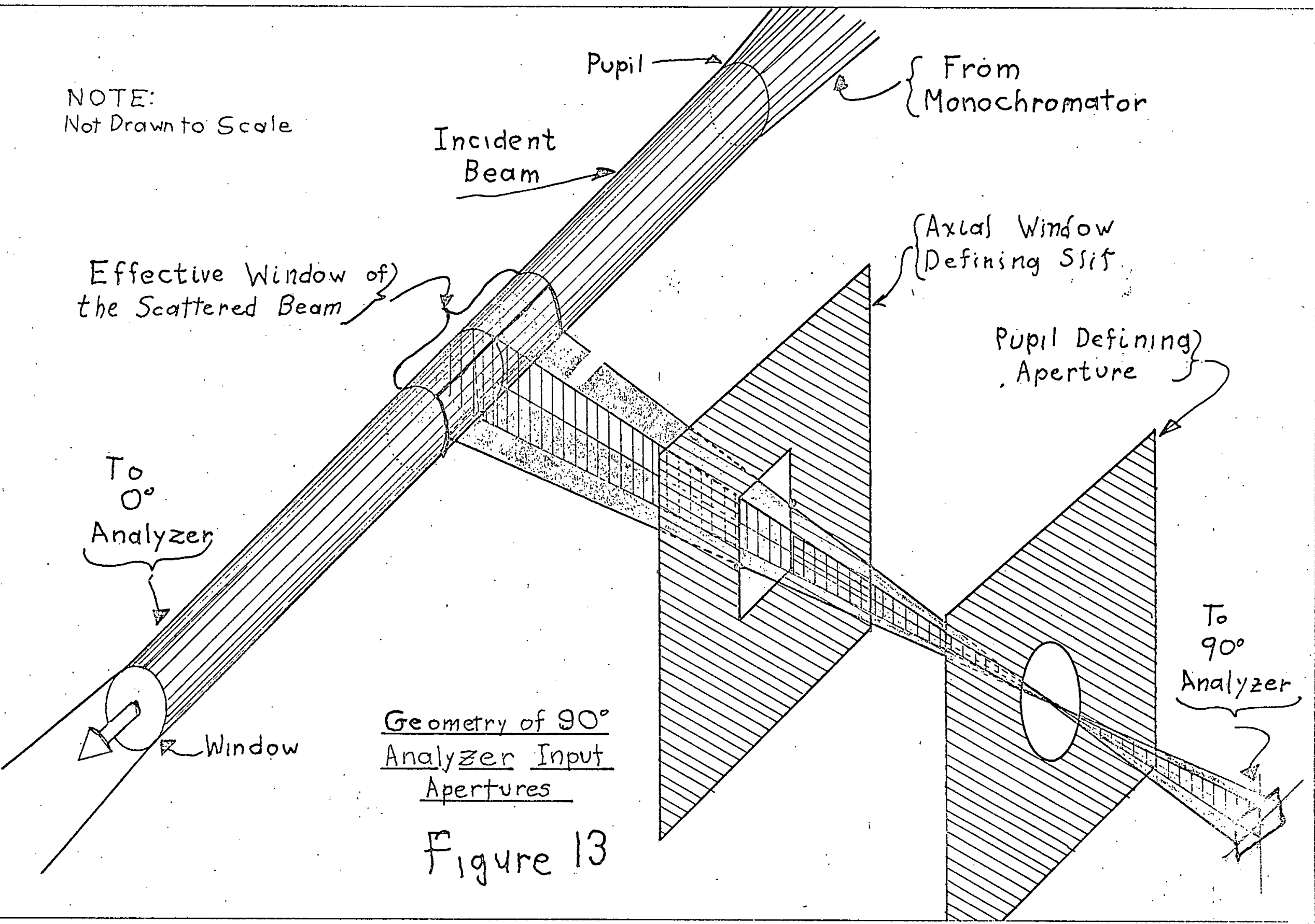


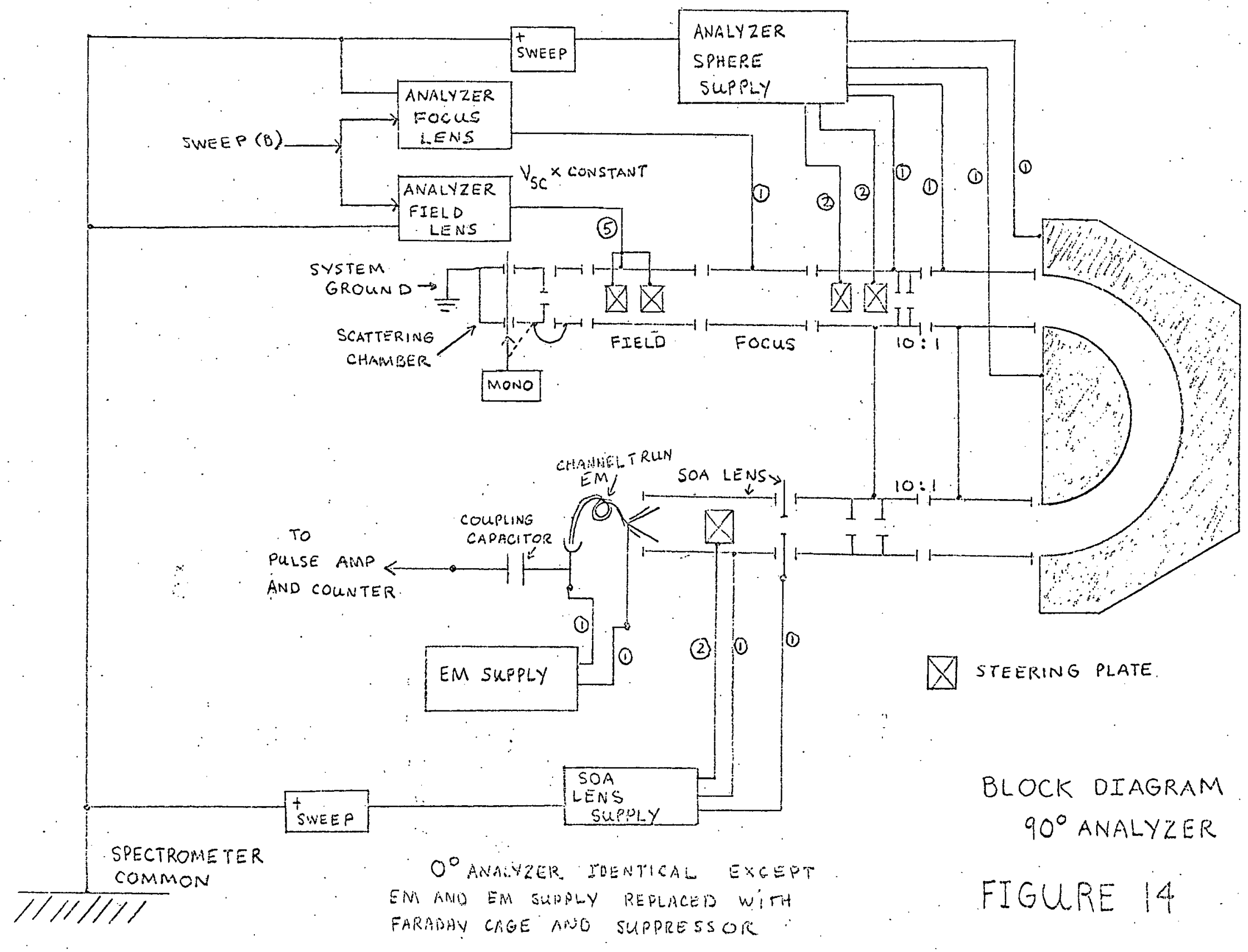




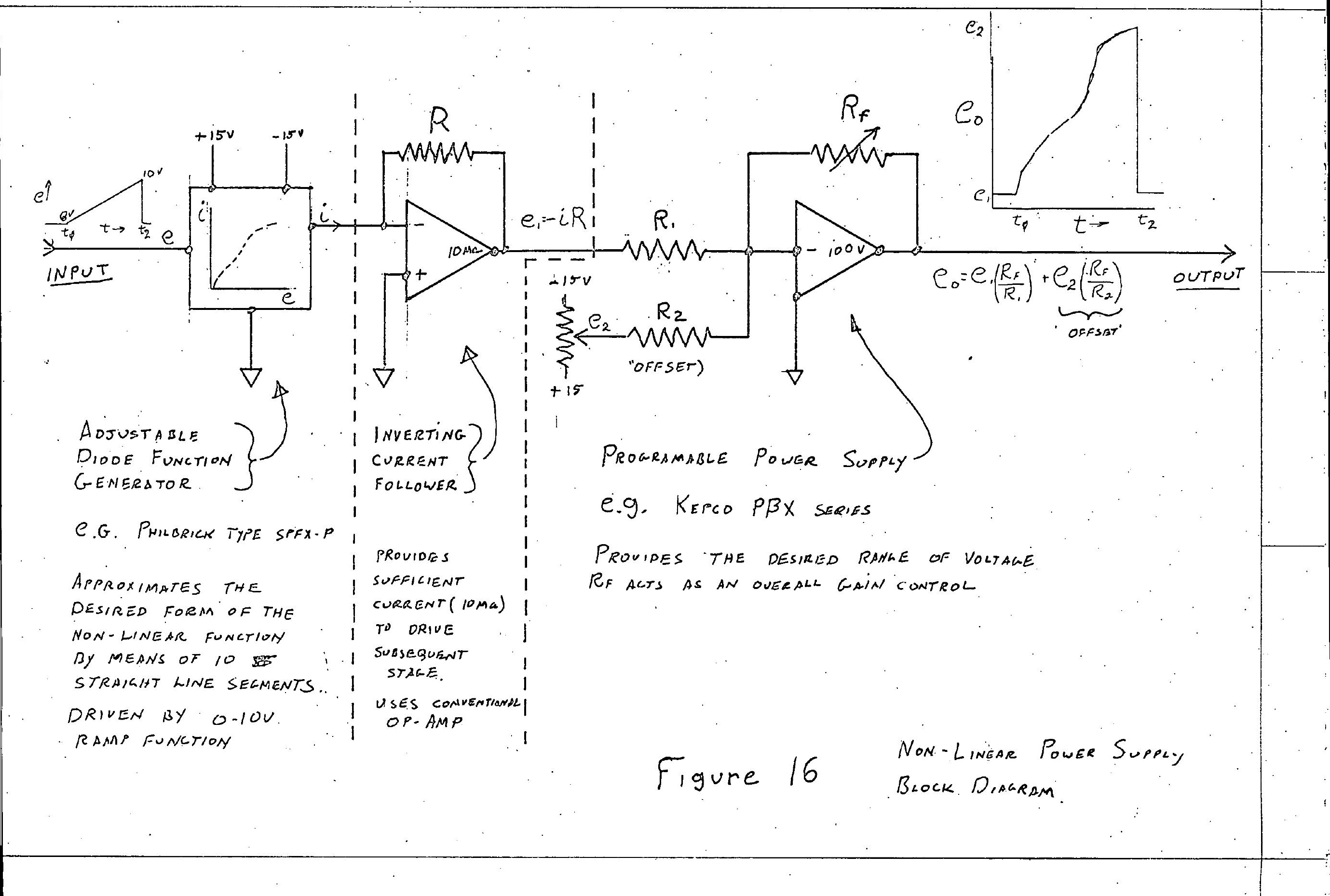




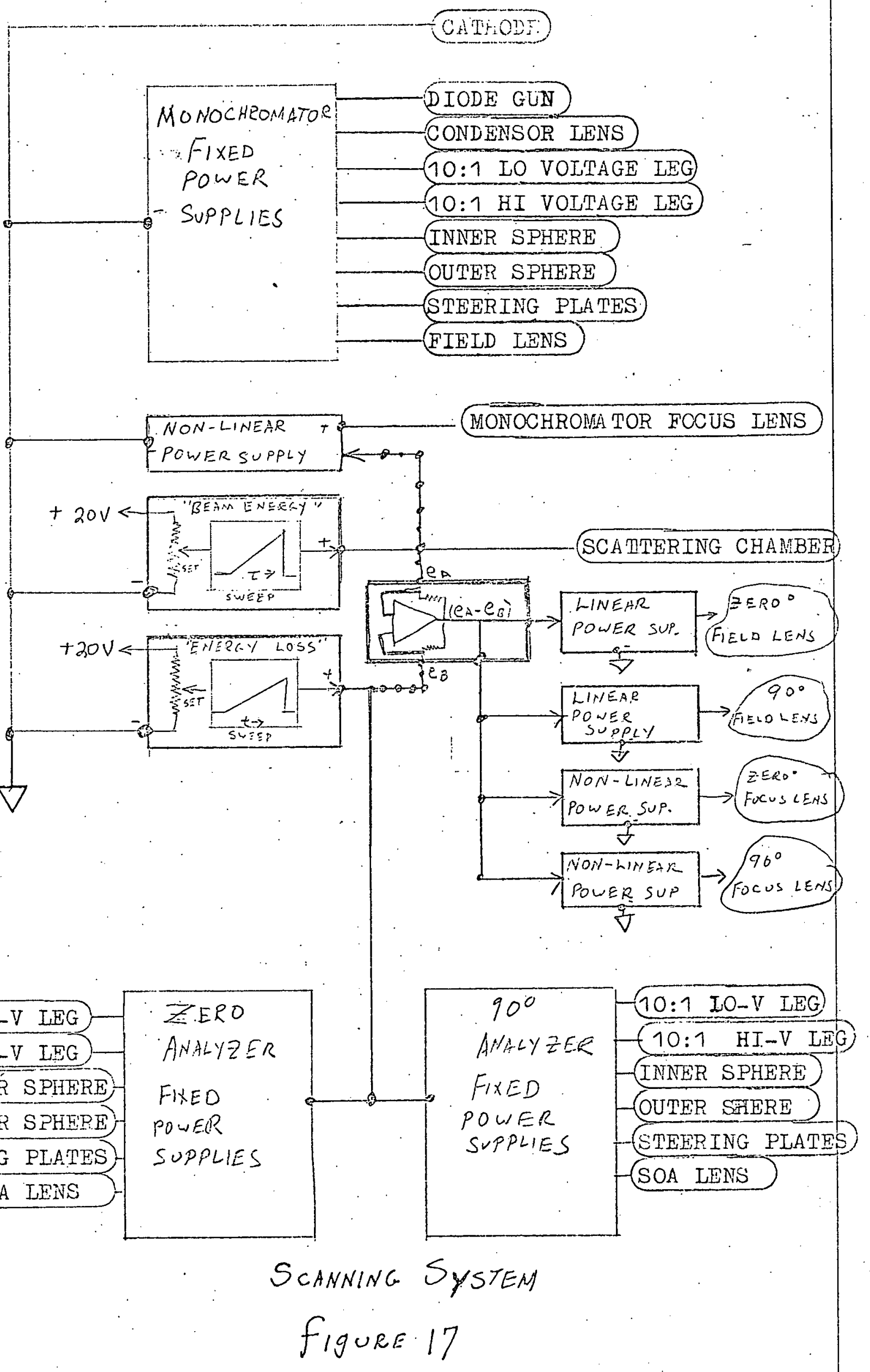




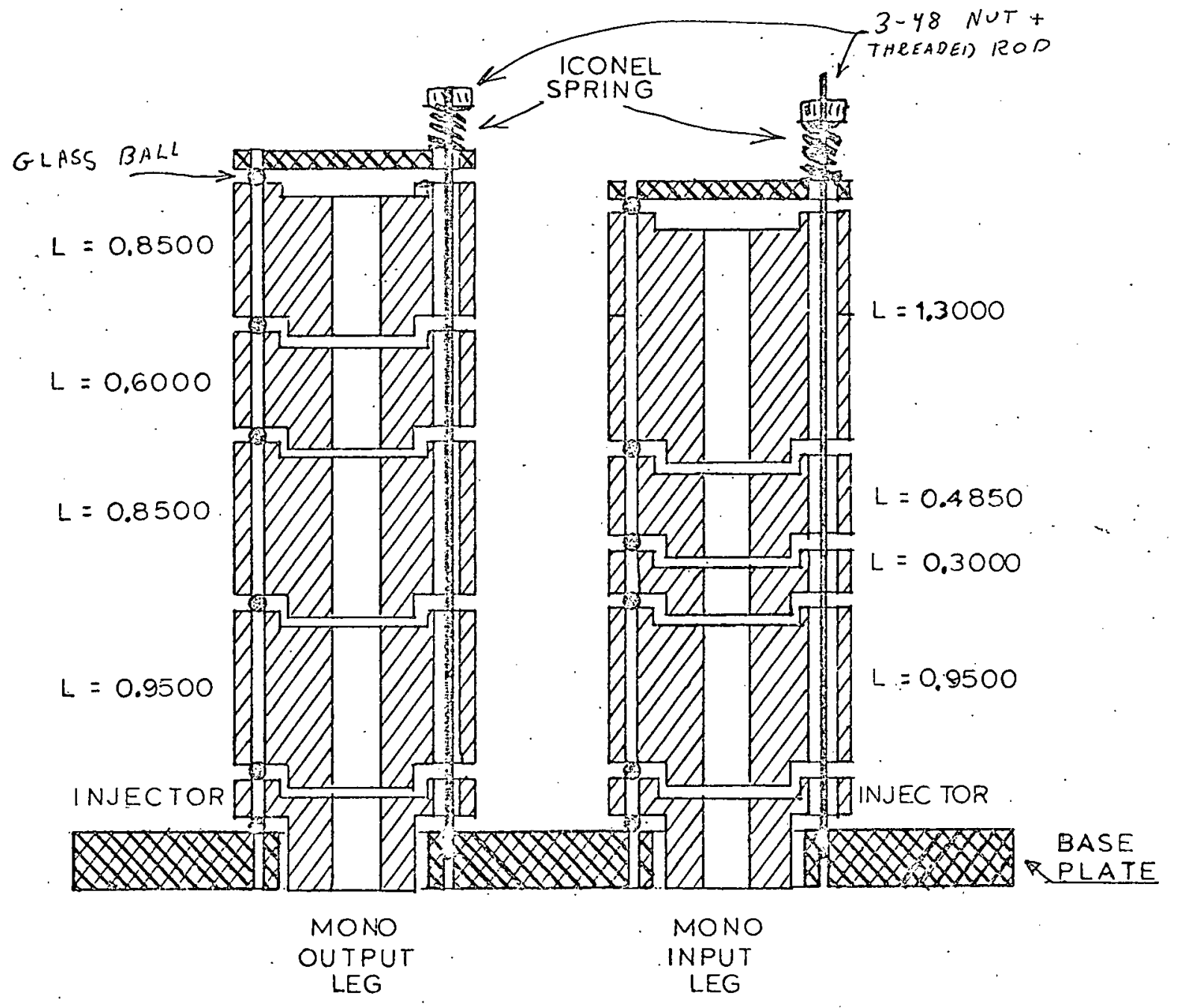

$\frac{\text { MODEL }}{\text { FIGURE } 18}$

$12-12-68$

BRAWING-4. \# 


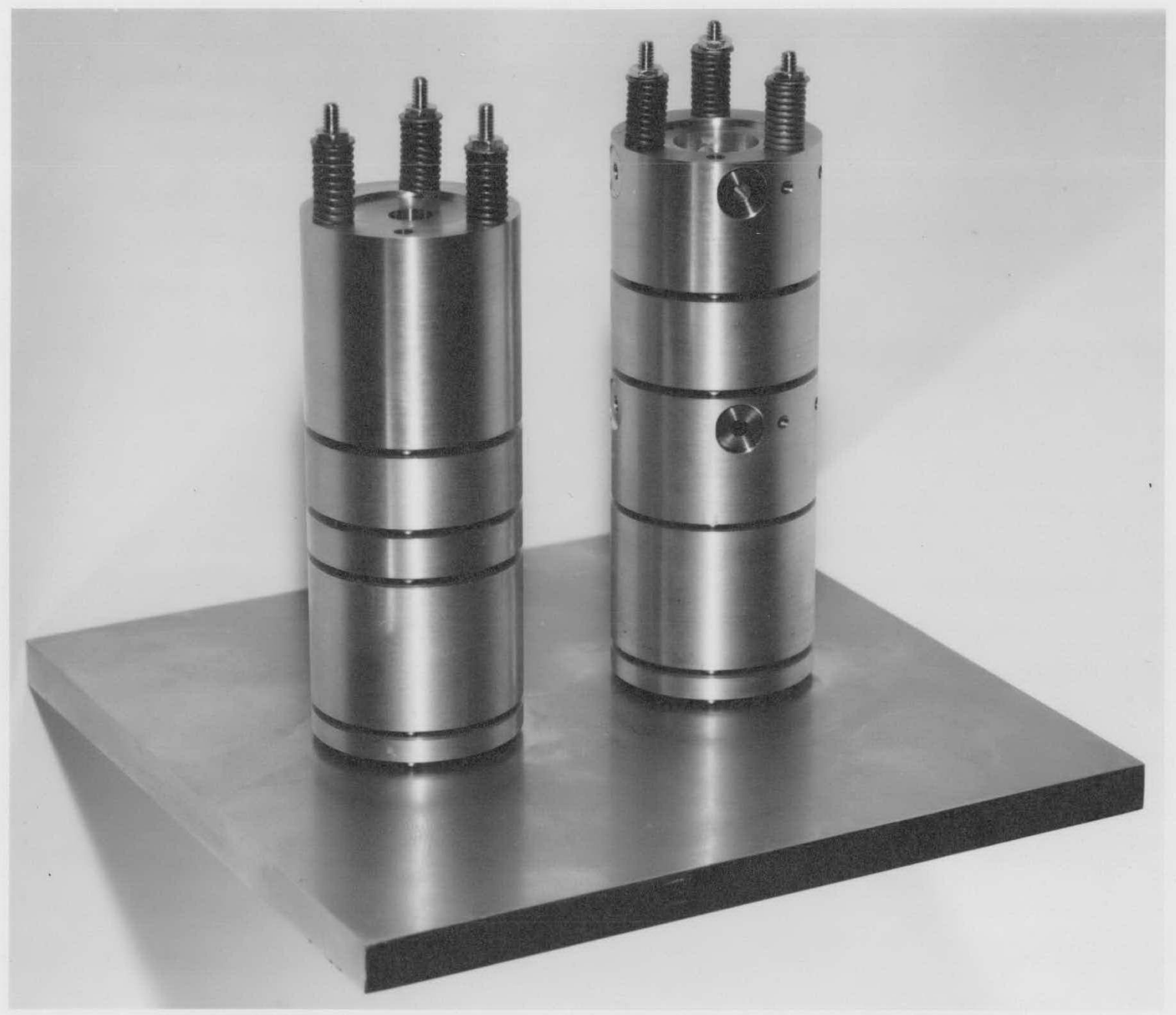

$$
F_{19} \cdot 18 a
$$

